Ann. Biol. anim. Bioch. Biophys., I962, 2 (I), I7-23.

\title{
NOUVELLES DONNÉES SUR LA TRANSPLANTATION DES EUFS, CHEZ LA LAPINE, PAR VOIE VAGINALE OU INTRAPÉRITONÉALE
}

\author{
L. DAUZIER \\ avec la collaboration technique de Elyane Goussopoutos \\ Laboratoire de Zootechnie, Centre de Recherches agronomiques du Midi, \\ École nationale supérieure agronomique, Montpallier (Hérault).
}

\section{SOMMAIRE}

La transplantation des œufs chez les femelles domestiques n'avait pu être réussie par voie vaginale ou intrapéritonéale.

Au cours d'un travail réalisé sur I4I Lapines et I 080 œufs nous avons pu obtenir le développement de 89 œufs sur I9I œufs transplantés par perforation de la paroi vaginale (46,6 p. 100), de 75 œufs sur 444 œufs transplantés sans perforation de la paroi vaginale ( 7 p. IO0) et de I 3 sur I4I œufs inovulés par voie intrapéritonéale (9,2 p. I00).

En opérant par voie vaginale, les pertes d'cufs se situeraient à deux stades :

- dès leur dépôt dans les cornes utérines la moitié d'entre eux seraient perdus, vraisemblablement par expulsion dans le vagin;

- le deuxième stade se situerait après la répartition des œufs le long de la corne utérine.

\section{INTRODUCTION ETT BIBLIOGRAPHIE}

Chez les animaux domestiques, l'inovulation sans intervention chirurgicale, qui faciliterait. la transplantation des œufs soit à des fins expérimentales, soit dans certains élevages, peut notamment se concevoir par voie vaginale ou intrapéritonéale. Or les auteurs qui ont abordé ce problème, ont enregistré des échecs en déposant les œufs dans le tractus génital de la femelle receveuse par voie vaginale (DowI,ING, I949; Rowson, I95I ; Willet, I953 ; DZIUK, DONKER, Nichols, PETERSEN, I958 ; BeATTTY, I95I, ayant seul obtenu le développement de 5 œufs chez la Souris), soit intrapériritonéale (HAFEZ, I96I). C'est ce qui nous a amené à reprendre cette étude chez la Lapine, pour préciser dans quelle mesure le transfert des oufs, par les voies précitées ne peut être réalisé. 


\section{MATÉRIEL, ET MÉTHODESS}

Ces expériences ont porté sur I4 I Lapines et I 080 œufs ou blastocystes.

\section{$\mathrm{I}^{\circ}$ Inovulation par voie vaginale.}

La nécessité de déposer les ceufs dans l'utérus d'une part, et d'avoir une synchronisation entre le stade de développement de l'œuf et l'état physiologique de l'utérus de la Lapine receveuse d'autre part, nous ont conduit à utiliser des blastocystes de 4 jours et demi. En effet, CHANG (I950), NoyEs et DICKMANN (I960) ont montré que les chances de réussite de transplantation des oufs in utéro, chez la Lapine, étaient les plus grandes quand cette synchronisation était respectée. Or, ceux-ci n'atteignent les cornes utérines que 62 à 82 heures après l'ovulation (PINCUs, 1930).

Les blastocystes obtenus par perfusion des cornes utérines avec une solution physiologique (une partie de sérum homologue chauffé à $56^{\circ} \mathrm{C}$ pendant $3^{\circ} \mathrm{mn}$, une partie de solution de Locke ; Streptomycine : I ooo $\gamma / \mathrm{ml}$ de solution finale) sont conservés in vitro quelques minutes à une température voisine de $35^{\circ} \mathrm{C}$ avant leur dépôt dans les cornes utérines de la Lapine receveuse.

Le transfert des blastocystes par voie vaginale est réalisé après laparatomie de deux façons différentes :

- Soit par perforation de la paroi vaginale : la pointe de la micropipette est guidée, après perforation de la paroi du vagin, dans le cervix, puis dans les cornes utérines où sont déposés les ceufs.

- Soit sans perforation de la paroi vaginale : la pointe de la micropipette est introduite jusqu'à l'entrée du cervix, dans la lumière d'un tube de verre faisant office de spéculum (tube préalablement introduit dans le vagin par voie vulvaire) puis guidée, comme précédemment, dans la corne utérine. Les opérations sont dans ce demier cas comparables à celles effectuées, avec spéculum, chez la Vache par d'autres auteurs sans toutefois de laparatomie (Willet i953, Dzilk, Donker, Nichols, PeterSEN I958). tocystes.

Les Lapines sont sacrifiées, sauf précisions contraires, 12 jours après la transplantation des blas-

\section{$2^{\circ}$ Inovulation par voie intrapéritonéale}

Les cufs fécondés, récupérés 4 heures après la ponte ovulaire, ont été injectés dans la cavité générale de Lapines accouplées quelques minutes auparavant avec un mâle vasectomisé.

L'ovulation se produisant environ 12 heures après le coït, il y a donc un déphasage de 16 heures entre les Lapines donneuses et receveuse.

Le dépôt des œufs est effectué de la façon suivante : la paroi abdominale est perforée latéralement au niveau de la région ovarienne, avec un trocart, dans la lumière duquel est introduite une micro* pipctte contenant les cufs, qui sont ensuite expulsés dans la cavité abdominale. Les Lapines sont dans ce cas sacrifiées 24 heures après le dépôt des cufs.

\section{RÉSULTATS}

\section{I $^{\circ}$ Inovulation par voie vaginale}

a) Avec perforation de la paroi vaginale:

L'ensemble des résultats est rapporté au tableau I : 46,6 p. Ioo des œufs se sont développés dans $84,6 \mathrm{p}$. Ioo des Lapines utilisées.

TABLEAU I

\begin{tabular}{c|c|c|c}
\hline Nombre de lapines & $\begin{array}{c}\text { Nombre de lapines } \\
\text { gestantes }\end{array}$ & $\begin{array}{c}\text { Nombre de blastocystes } \\
\text { transplantés }\end{array}$ & $\begin{array}{c}\text { Nombre de blastocystes } \\
\text { implantés }\end{array}$ \\
\hline 26 & $22(84,6 \%)$ & 191 & $89(6,6 \%)$ \\
\hline
\end{tabular}


b) Sans perforation de la paroi vaginale:

Les résultats sont dans ce cas nettement plus faibles, aussi bien en ce qui concerne les Lapines gestantes, que les blastocystes implantés.

TABIEAU 2

\begin{tabular}{|c|c|c|c|}
\hline Nombre de lapines & $\begin{array}{c}\text { Nombre de lapines } \\
\text { grestantes }\end{array}$ & $\begin{array}{c}\text { Nombre de blastocystes } \\
\text { transplantés }\end{array}$ & $\begin{array}{c}\text { Nombre de blastorystes } \\
\text { implantés }\end{array}$ \\
\hline $1 i 3$ & $21(33,3 \%)$ & is's & $75(17 \%)$ \\
\hline
\end{tabular}

c) Influence du nombre d'xuts déposés:

Il ressort du tableau 3 que le nombre d'œufs déposés affecte, ce qui parait logique, le nombre de Lapines gestantes quelle que soit la technique utilisée. Par contre, le nombre de blastocystes qui évoluent ne paraît nettement influencé qu'en opérant sans perforation de la paroi vaginale.

TABI,EAU 3

\begin{tabular}{|c|c|c|c|c|c|c|c|}
\hline Transfert & \multicolumn{3}{|c|}{ avec perforation de la paroi vaginale } & \multicolumn{4}{|c|}{ Transfert sans perforation de la paroi vaginale } \\
\hline Lapines & gestantes & Blastocystes & inplantes & Japines & gestantes & Blastocyste & implantés \\
\hline $\begin{array}{c}<8 \text { \&ufs } \\
\text { déjoosés }\end{array}$ & $\begin{array}{c}\geqslant 8 \text { œufs } \\
\text { déposés }\end{array}$ & $\begin{array}{l}<8 \text { oeufs } \\
\text { déposés }\end{array}$ & $\begin{array}{l}\geqslant 8 \text { aufs } \\
\text { déposés }\end{array}$ & $\begin{array}{c}<8 \alpha u f s \\
\text { déposés }\end{array}$ & $\begin{array}{l}\geqslant 8 \text { oxufs } \\
\text { déposés }\end{array}$ & $\begin{array}{l}<8 \text { oufs } \\
\text { déposés }\end{array}$ & $\begin{array}{c}\geqslant 8 \text { aenfs } \\
\text { déposés }\end{array}$ \\
\hline$\cdots-\cdots-$ & $\cdots$ & --- - & $\cdots-\cdots$ & - & $-\cdots$ & --- & $-\ldots-\ldots$ \\
\hline $\begin{array}{c}10 / 13 \\
(76,9 \%)\end{array}$ & $\begin{array}{c}12 / 13 \\
(92,3 \%)\end{array}$ & $\begin{array}{c}28 / 633 \\
(4+4,4 \%)\end{array}$ & $\begin{array}{l}(51 / 12 k \\
(47,6 \%)\end{array}$ & $\begin{array}{l}8 / 38 \\
(21 \%)\end{array}$ & $\begin{array}{l}13 / 25 \\
(52 \%)\end{array}$ & $\begin{array}{c}13 / 206 \\
(5,3 \%)\end{array}$ & $\begin{array}{l}62 / 238 \\
(26 \%)\end{array}$ \\
\hline
\end{tabular}

d) Influence du niveau des cornes utérines où les ceufs sont déposés:

On pouvait penser que, dans les conditions naturelles les œufs se trouvant vers le sommet des cornes utérines au moment où nous avons opéré, par rapport à l'ovulation, le niveau des cornes utérines, où les blastocystes étaient déposés, pourrait intervenir ; en fait il n'en est rien.

TABLEAU 4

\begin{tabular}{|c|c|c|c|c|c|}
\hline Transfert & avec perfora & on de la praroi raginale & \multicolumn{3}{|c|}{ Transfert sans perforation de la paroi vaginale } \\
\hline Lapines & gestantes & Blastocystes implantés & Lapines gestantes & Blastocystes & implantés \\
\hline \multicolumn{6}{|c|}{ Niveau des cormes utérines oì les Blastocystes ont été déposés } \\
\hline Bas & Haut & Haut & Haut & Bas & Haut \\
\hline $\begin{array}{c}16 / 19 \\
(8 \%, 2 \%)\end{array}$ & $\begin{array}{c}6 / 7 \\
(85,7 \%)\end{array}$ & $\begin{array}{c}2-2 / 13 \\
(51,1 \%)\end{array}$ & $\begin{array}{c}12 / 34 \\
(35,3 \%)\end{array}$ & $\begin{array}{c}38 / 220 \\
(17,2 \%)\end{array}$ & $\begin{array}{c}37 / 22 \% \\
(16,5 \%)\end{array}$ \\
\hline
\end{tabular}


e) Influence du nombre de corps jaunes de la Lapine receveuse:

Contrairement à ce qu'a observé Rowsor chez la Brebis en opérant avec des œufs généralement au stade 8-ıo blastomètres, la transplantation des blastocystes de Lapine ne donne pas, dans les conditions de nos essais, de meilleurs résultats quand le nombre de corps jaunes de la Lapine receveuse est supérieur au nombre d'œuf déposés.

TABIEAU 5

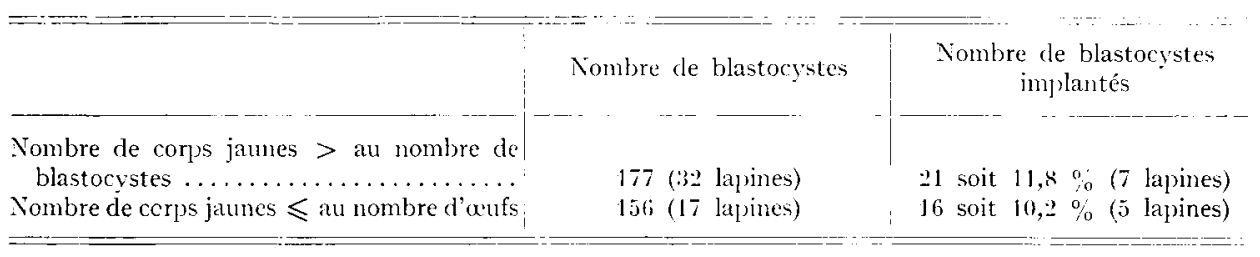

\section{f) Localisation des blastocystes:}

Les très mauvais résultats obtenus en opérant sans perforation de la paroi vaginale posent le problème de la disparition des blascotystes.

C'est ainsi que 1'on constate (tableau 6) que près de la moitié des blasctocystes ne sont pas trouvés dans les cornes utérines de femelles receveuses sacrifiées de $45 \mathrm{mn}$ à 2 heures après leur transfert.

\section{TABIEAU 6}

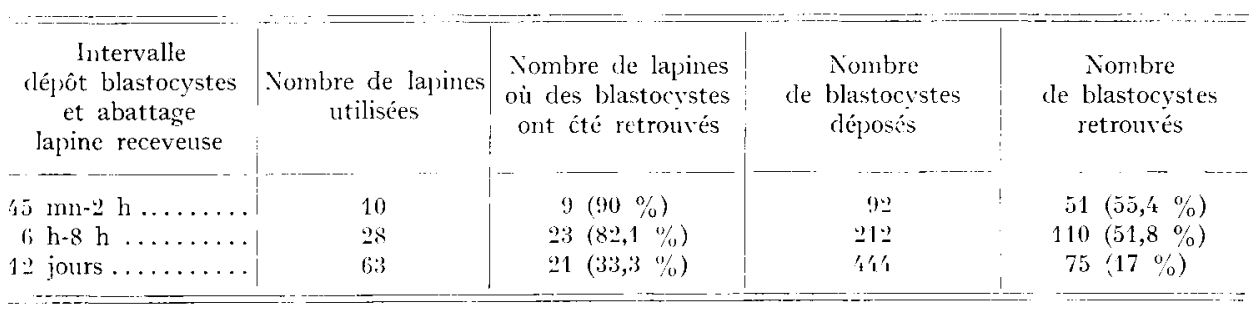

Une étude plus précise a même montré que dans les 2 heures suivant l'opération, la grande majorité des blastocystes trouvés étaient présents dans la moitié inférieure des cornes utérines (tableau 7), un certain nombre d'entre eux étant dans le vagin.

On constate par la suite une équirépartition des œufs au sein de la corne utérine et une diminution progressive du nombre de blastocystes implantés.

TABLEAU 7

\begin{tabular}{|c|c|c|c|c|c|}
\hline \multirow{2}{*}{$\begin{array}{c}\text { Intervalle } \\
\text { dépoôt blastocystes } \\
\text { et abattage } \\
\text { lapine receveuse }\end{array}$} & \multirow{2}{*}{$\begin{array}{l}\text { Nombre } \\
\text { de blastocystes } \\
\text { déposés }\end{array}$} & \multirow{2}{*}{$\begin{array}{l}\text { Nombre } \\
\text { de blastocystes } \\
\text { trouvés }\end{array}$} & \multicolumn{3}{|c|}{$\begin{array}{l}\text { Nombre d'oufs retrouvés } \\
\text { à différents niveaux du tractus }\end{array}$} \\
\hline & & & Vagin & $\begin{array}{l}\text { Moitié inférieure } \\
\text { des cornes utérines }\end{array}$ & $\begin{array}{l}\text { Moitié supérieure } \\
\text { des cornes utérines }\end{array}$ \\
\hline$t_{15}$ & 97 & 45 (1: lapin & 6 & $4:$ & 0 \\
\hline $6 \mathrm{~h}-8 \mathrm{~h} \ldots$ & 71 (8 lapines) & 32 (6 lapines) & 0 & $2:$ & 10 \\
\hline
\end{tabular}




\section{$2^{\circ}$ Inovulation par voie intrapéritonéale}

Il ressort du tableau 8 qu'il est possible d'obtenir le développement d'œufs dans dans le tractus génital de Lapines qui ont reçı ceux-ci par voie intrapéritonéale.

\section{TABIEAU 8}

\begin{tabular}{|c|c|c|c|}
\hline $\begin{array}{c}\text { Tombre de lappines } \\
\text { utilisées }\end{array}$ & $\begin{array}{l}\text { Nombre de lapines } \\
\text { où les aufs transplantés } \\
\text { ont été trouvés } \\
\text { dans le tractus génital }\end{array}$ & $\begin{array}{l}\text { Nombre d'oeufs } \\
\text { transplantés }\end{array}$ & $\begin{array}{c}\text { Nombre d'oufs trouvés } \\
\text { dans le tractus génital } \\
\text { de lapine receveuse }\end{array}$ \\
\hline 14 & $6(42,8 \%)$ & $1: 1$ & $13(9,2 \%)$ \\
\hline
\end{tabular}

Bien que les résultats ne soient pas excellents, les résultats positifs obtenus ne semblent guère prêter à discussion (tableau 9) tous les cufs vierges pondus par les Lapines receveuses ont été trouvés, les œufs en surnombre, seuls segmentés, ne pouvant donc résulter que de la transplantation. Par ailleurs, aucune lésion du tractus génital n'a été observée ce qui exclut la possibilité d'un dépôt direct des oufs dans l'appareil génital au cours des manipulations.

TABI,EAU 9

\begin{tabular}{|c|c|c|c|c|}
\hline To lapines & $\begin{array}{c}\text { Nombre d'wufs } \\
\text { pondus par la lapine } \\
\text { receveuse (nombre } \\
\text { corps james) }\end{array}$ & $\begin{array}{l}\text { Nombre d'oufs } \\
\text { vierges trouvés }\end{array}$ & $\begin{array}{l}\text { Nombre d'oufs } \\
\text { transplantés }\end{array}$ & $\begin{array}{l}\text { Nombre d'œufs } \\
\text { segrmentés trouvés }\end{array}$ \\
\hline 90 & 10 & 10 & 8 & 3 \\
\hline 121 & 16 & 16 & 11 & 1 \\
\hline 179 & 9 & 9 & 9 & 1 \\
\hline 207 & 11 & 11 & 9 & 1 \\
\hline 230 & 0 & 6 & 9 & 4 \\
\hline 190 & () & 0 & 11 & 3 \\
\hline
\end{tabular}

On peut même constater qu'un résultat positif a été obtenu avec une Lapine receveuse qui n'avait pas ovulé au moment de l'abattage.

\section{DISCUSSION}

Ces résultats montrent nettement que contrairement à ce que prétendent les autres auteurs, il est possible de transférer avec succès des œufs soit par voie vaginale soit par voie intrapéritonéale.

Les conditions de succès ne sont pas suffisamment connues pour espérer utiliser couramment cette technique, mais ces résultats appellent quelques remarques:

S'il paraît difficile d'émettre une hypothèse sur les différences des résultats constatées que l'on opère ou non par perforation vaginale, il semble toutefois que les pertes d'œufs se situent à différents stades. 
Tout d'abord comme l'ont signalé Rowson, HARPER, BENNETT (I96I, communication personnelle transmise par EDWARDS) qui ont opéré avec des particules radioactives, il y a, dès le dépôt des ouffs dans l'utérus, une perte importante de ceux-ci, perte qui pourrait être en rapport avec leur élimination dans le vagin. En effet, si nous n'avons retrouvé qu'un petit nombre de blastocystes dans le vagin, il faut préciser que leur recherche dans le liquide de perfusion est très difficile, celui-ci étant presque toujours souillé par du sang ou de l'urine. Ces pertes correspondent à près de la moitié des œufs déposés.

Mais il y a par la suite, peut être du fait des réactions utérines, une dégénérescence des oufs qui semble se situer après la répartition des œufs le long de la corne utérine.

Rappelons à ce sujet que Brock et Rowson (I952) attribuent les échecs antérieurs à une faible résistance à l'infection de l'utérus en phase lutéinique.

En ce qui concerne les résultats obtenus par voie intrapéritonéale, il est bien évident que restent à préciser les conditions dans lesquelles le tropisme dirigeant l'œuf vers les trompes se produit car il faut rappeler que dans l'étude ci-dessus il y a un déphasage d'environ 16 heures entre les Lapines donneuse et receveuse.

Ces résultats sont à rapprocher de ceux de LEOPOLD (I880) qui, après enlèvement d'un ovaire et ligature de la trompe opposée, observait le passage des œufs de Lapines pondus par l'ovaire restant dans la trompe non ligaturée. On peut également rappeler que les spermatozoïdes déposés par voie intrapéritonéale peuvent féconder les cufs dans les trompes de Fallope (DaUzier, 'Thibaui,T, I956).

\section{Rę̧u en décembre 1961}

\section{SUMMARY}

NEW FACTS CONCERNING THE TRANSPLANTATION OF LGGS, IN THE RABBIT, VAGINALLY OR INTRAPERITONEALLY

The transplantation of eggs in domestics females had never been successful vaginally or intraperitoneally.

In the course of a study carried out on I4I rabbits and Io80 eggs we have succeeded in oobtaining the development of 89 out of IgI eggs transplanted by perforation of the vaginal wall (46.6 p. 100), of 75 out of 444 eggs transplanted without perforation of the vaginal wall ( $\mathrm{I} 7 \mathrm{p}$. 100 ) and of $\mathrm{I} 3 \mathrm{out}$, of 141 eggs inovulated intraperitoneally ( $9.2 \mathrm{p}$. 100).

Operation vaginally, the loss of eggs takes place at two stages :

- as soon as they are deposited in the uterine horn half of them seem to be lost, probably by expulsion into the vagina.

- the second stage is after the distribution of ths eggs along the uterine horn.

\section{RÉFÉRENCES BIBLIOGRAPHIQUES}

Beatty R. A., 1951. Transplantation of Mouse of eggs. Nature, 168, 995.

Brock H., Rowson L. E., I952. The production of viable Bovine ova. J. Agr. Sci., 42, 479-482.

Chang M. C., I950. Development and fate of transfered Rabbit ova or blastocyst in relation to the ovulation time of recepients. J. Exp. Zool., 114, I97-226.

Dauzier L., Thibault C., I956. Recherche expérimentale sur la maturation des gamètes máles chez les Mammifères, par l'étude de la fécondation "in vitro de l'œuf de Lapine". Proc 3rd internat. Cong. Aninn. Reprod. Cambridge. 
Dowling D. F., 1949. Problems of the transplantation of fertilized ova. J. Agr. Sci., 39, 374-396.

Dziuk P. J, Donker J. D., Nichols J. R., Petersen W. E., I958. Problems associated with the transfer of ova between. Cattle. Univ. Minn. Agr. Expt. Stat. Techn. Bull., 222, 75 pp.

HAFEZ E. S. E., I961. "In vivo" and "in vitro" studies on Rabbit ova ; non surgical ova transfer as a target. Proc. 4th internat. Cong. Anim. Reprod. La Haye.

L.Eopold G., I88o. Die Ueberwanderung der Eier - Line experimentelle Studie. Arch. \%. Gynäk, 16, $24-44$. Noyes R. W., Dickmane 7., i960. Relationship of ovular age to endometrial development. J. Reprod. Fertil., 1, 186-196.

Pincus G., I930. Proc. Roy. Soc. London, 107, B, I32-167.

Rowson L. E., r951. Methods of inducing multiple ovulation in Cattle. J. Endocrinol., 7, 260-270.

Rowson L. E., Harper M. J., Bennett J. P., i960. Totes on ovum transfer in Cattle. "Comminication personnelle " transmise par EDWARDS.

Willet E. L., 1953. Fgg transfer and superovulation in farm animals. Iowa State Col., J. Sci., 28 :83-100. 